\title{
Application of an Iliac Osteocutaneous and Fabricated Chimeric Iliac Osteocutaneous Flap for Foot and Ankle Reconstruction
}

\author{
Zhao Hui Pan, MD ${ }^{1}$ Ping Ping Jiang, MD ${ }^{1}$ Shan Xue, MD ${ }^{1}$ jian Li Wang, MD \\ ${ }^{1}$ Department of Orthopaedics, Institute of Orthopaedic Trauma \\ Surgery of Chinese People's Liberation Army, Weifang, China \\ Address for correspondence Zhao Hui Pan, MD, Institute of \\ Orthopaedic Trauma Surgery of the Chinese People's Liberation Army, \\ 89th Hospital, 256 Beigongxijie, Weifang, Shandong Province, China \\ J Reconstr Microsurg Open 2017;2:e111-e117. \\ 261021 (e-mail: panzhaohui89yy@hotmail.com).
}

\begin{abstract}
Keywords

- iliac osteocutaneous flap

- superficial circumflex iliac artery

- fabricated chimeric flap

- foot and ankle

- complex bone defect

Background As understanding of the blood supply by superficial circumflex iliac artery (SCIA) to the skin and iliac bone has improved and the use of a perforator flap has become accepted, most previous drawbacks of SICA iliac osteocutaneous flaps, such as bulky flap, small diameter, and inadequate blood supply to bone, can now be overcome. Here, the authors present their experience of using such flaps for the reconstruction of complex defects in the foot and ankle with a focus on feasibility and safety.

Methods A retrospective review of patients who underwent foot and ankle reconstruction using an SCIA iliac osteocutaneous flap between 2010 and 2015 was performed to assess outcomes.

Results Four patients who underwent treatment with SCIA iliac osteocutaneous flaps and eight patients treated with fabricated chimeric iliac osteocutaneous flaps were identified. The iliac segment size ranged from $1 \times 3 \times 0.7$ to $3 \times 6 \times 1 \mathrm{~cm}$ and the skin paddle size ranged from $1 \times 4$ to $8 \times 16 \mathrm{~cm}$. All flaps survived uneventfully except for marginal necrosis in one anterolateral thigh (ALT) flap and one iliac osteocutaneous flap. The median time to bone union was 4 months. All patients were able to walk in normal footwear and none developed significant complications at the donor site.

Conclusion The use of free SCIA iliac osteocutaneous and fabricated chimeric iliac osteocutaneous flaps provides an alternative for treating small- and medium-sized bone defects (smaller than $8 \mathrm{~cm}$ ) along with soft tissue defects in the foot and ankle region.
\end{abstract}

In the foot and ankle region, the use of vascularized bone flaps is usually adequate for managing complex bone defects. ${ }^{1}$ Flaps from various donor sites, such as the fibula, deep circumflex iliac artery-iliac crest, and medial femoral condyle, have been explored, ${ }^{1-7}$ with each type of flap showing positive and negative characteristics. More work is needed to identify an ideal donor site that can improve functional and aesthetic results and decrease donor-site morbidity.

received

March 14, 2017 accepted after revision June 11, 2017
DOI https://doi.org/

10.1055/s-0037-1604341. ISSN 2377-0813.
The use of free superficial circumflex iliac artery (SCIA) iliac osteocutaneous flaps has been described for several decades. ${ }^{8}$ However, such flaps can be bulky, a drawback, which in combination with the small diameter of the SCIA, the variations that exist in pedicle anatomy, and the inadequate supply of blood to bone, has limited their widespread use for long bone reconstruction. As understanding of the blood supply by SCIA to the skin and iliac
Copyright $\odot 2017$ by Thieme Medical Publishers, Inc., 333 Seventh Avenue, New York, NY 10001, USA. Tel: +1(212) 584-4662.
License terms

(요 (1) $\odot$ 
bone has improved and the use of a perforator flap has become accepted, most of the above disadvantages can now be overcome. Recently, a free iliac osseous flap based on the periosteal branch of the SCIA was used in a case of maxillary reconstruction. ${ }^{9}$ We previously described performing hand reconstruction using an SCIA iliac osteocutaneous flap. ${ }^{10}$ Compared with other vascularized bone flaps, this type of flap is less invasive and less bulky. Additionally, this type of flap requires only a small volume of the iliac segment and is taken from an area with thin skin. Although SCIA iliac osteocutaneous flaps have several characteristics that are attractive for treating foot and ankle bone defects, their use for such purposes has not been reported to date.

In cases of extensive injury, a single osteocutaneous flap cannot easily cover an irregularly shaped, three-dimensional defect. In addition, when a potential recipient vessel is located far from a wound, the use of an osteocutaneous flap for microvascular anastomosis may be inadequate due to insufficient pedicle length. A combined flap may be necessary in these circumstances. Compared with other combination flaps, microsurgically fabricated chimeric osteocutaneous flaps can provide more tissue with better function. ${ }^{11}$ However, few studies on the use of fabricated chimeric osteocutaneous flaps for the reconstruction of complex bone defects in the foot and ankle region have been reported.

In this article, we reported our single-center experience of using an SCIA iliac osteocutaneous or a fabricated chimeric SCIA iliac osteocutaneous flap for foot and ankle reconstruction.

\section{Patients and Methods}

Following approval by the institutional review board of the Weifang 89th Hospital, a retrospective review was performed to assess operative outcomes of patients who underwent foot and ankle reconstruction using an SCIA iliac osteocutaneous flap between 2010 and 2015. Patients treated with other flap reconstruction or amputation were excluded. All procedures and examinations were performed by the same experienced surgeon. No cases were lost to follow-up. Plain radiographs were obtained at each visit. Foot function was measured based on American Orthopaedic Foot \& Ankle Society (AOFAS) foot score. ${ }^{12}$ The AOFAS score rates pain, function, and alignment using four grading scales (ankle-hindfoot, midfoot, hallux metatarsophalangeal-interphalangeal, and lesser metatarsophalangeal-interphalangeal scales), each of which has 100 possible points. Each patient's aesthetic satisfaction regarding the skin paddle was also evaluated using a selfreported visual analog scale (VAS) score with a range of 0 to 10 , with 10 indicating maximum satisfaction and 0 indicating minimum satisfaction. The scores were categorized as poor (0-2), fair (3-5), good (6-8), or excellent $(9-10) .^{13}$ Data for etiology, reconstructed site, flap component, size of graft, recipient vessel, complications, and outcome were collected.

\section{Surgical Procedures}

\section{Flap Design}

Flap design was chosen based on defect complexity, wound size, and recipient vessel status. Before surgery, a handheld Doppler probe was used to confirm the course of the SCIA and the locations of the perforators and periosteal branch of the deep branch of the SCIA. If there was ambiguity, the surgical plan was changed. Each flap was centered along the course of the deep branch of the SCIA and included at least one perforator. The medial border of the flap was placed lateral to the femoral triangle, and its lateral border could extend beyond the anterior-superior iliac spine (-Fig. 1A). A pinch test was performed to confirm primary closure of the donor site. For patients who underwent surgery to create a combined anterolateral thigh (ALT) flap, the ALT flap was designed from the ipsilateral side (-Fig. 1B).

\section{Operative Technique}

Thorough debridement was performed, and soft-tissue defect dimensions were measured. Recipient vessels were first isolated to confirm their patency and estimate pedicle length. Next, an incision was made from the superior border of the skin paddle to the anterior-superior iliac spine. In most cases, a superficial vein was identified and preserved. The subcutaneous tissue was subsequently divided superiorly and inferiorly until the periosteal branch of the SCIA was observed. The flap was then elevated in the lateral-to-medial
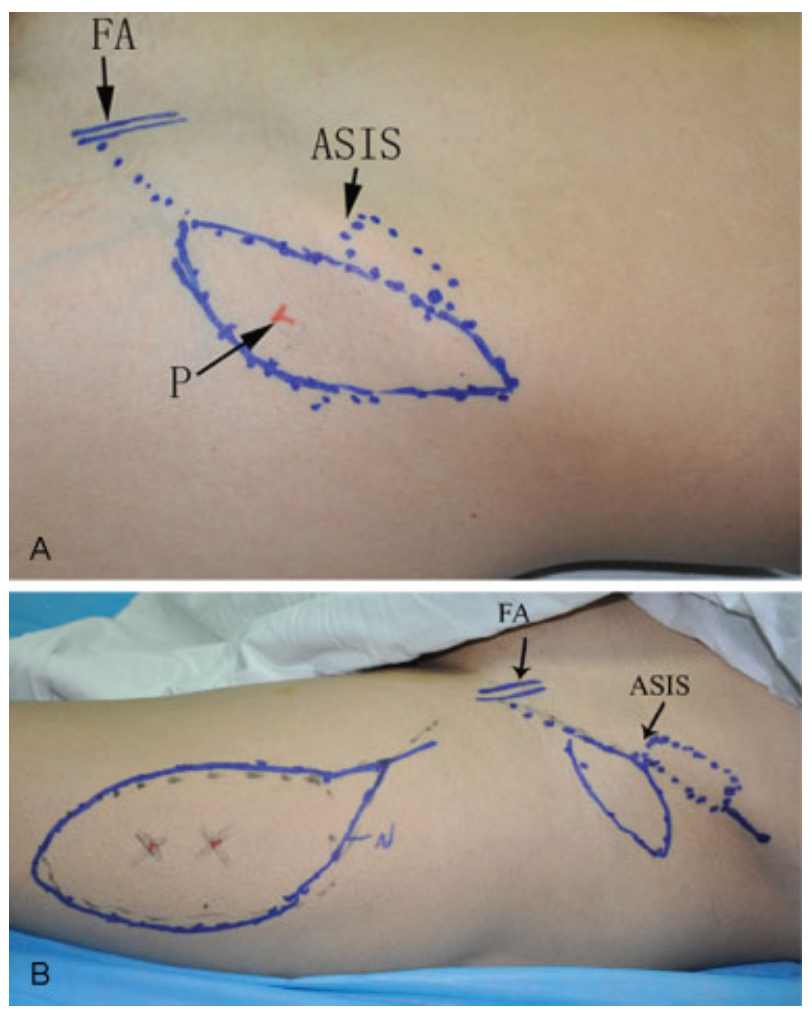

Fig. 1 Perioperative photographs demonstrating designs for two kinds of flaps. (A) Osteocutaneous and (B) Fabricated. ASIS, anterior-superior iliac spine; FA, femoral artery; $P$, perforator. 
direction from above the level of the deep fascia. Cutaneous perforators of the deep branch could usually be found $1 \mathrm{~cm}$ medial to the anterior-superior iliac spine. Because these perforators often cross the lateral femoral cutaneous nerve beneath the fascia, care was taken to avoid injuring the nerve. To protect the periosteal branch, a $0.5-\mathrm{cm}$ wide deep fascia cuff was left intact around each perforator prior to dissection. The outer surface of the iliac crest was then exposed by dividing the sartorius, tensor fasciae latae, and gluteus medius at their origins. Based on the direction of the periosteal branch, the required dimension of the iliac crest was harvested using an osteotome. Pedicle dissection was then performed in a retrograde manner, moving toward the femoral vessels. The deep fascia was incised to obtain a longer pedicle length and larger vessel diameter when

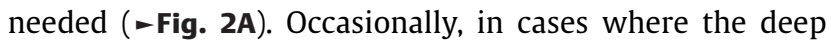
branch of the SCIA ran deep into the lateral femoral cutaneous nerve, the nerve had to be transected to preserve the continuity of the vessels. However, before closure of the iliac donor site, the nerve could be anastomosed if necessary. The integrity of the circulation was verified by inspecting the bleeding sites of both the bone graft and the flap ( - Video 1 ). The technique used for flap inset was tailored to the individual characteristics of each patient. The bone segment was either bridged into place or connected to the residual bone
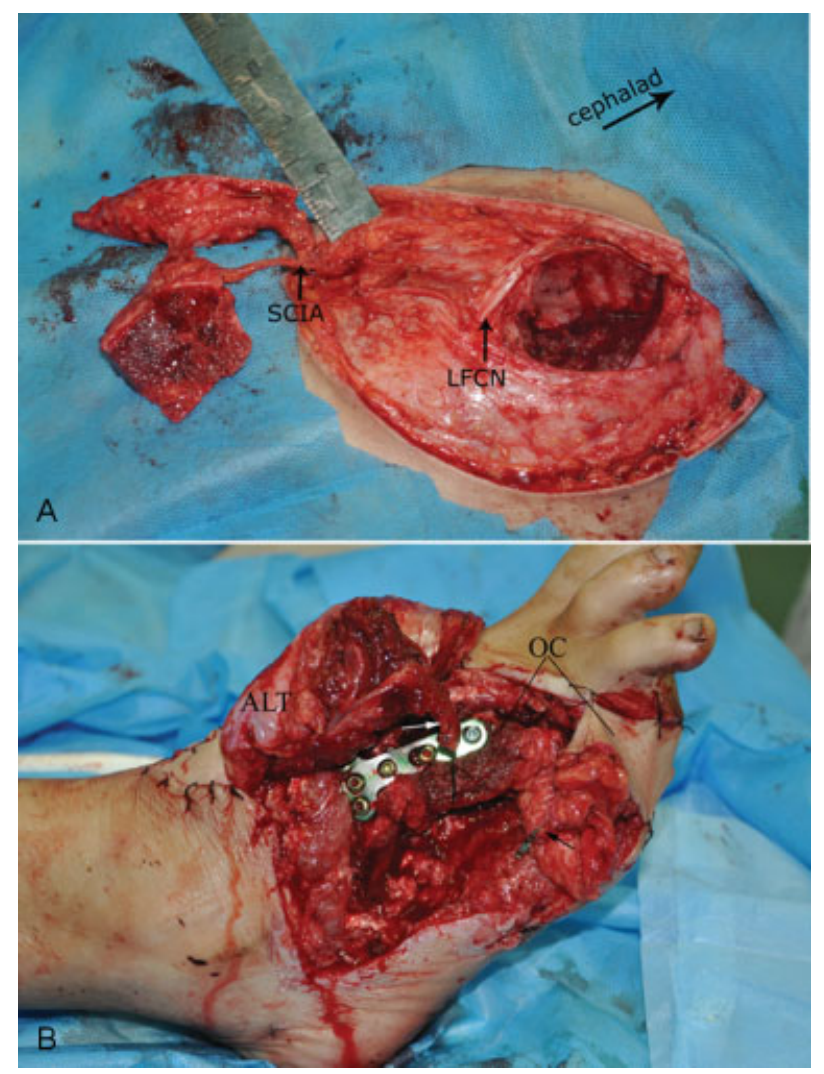

Fig. 2 Intraoperative photographs. (A) Elevated osteocutaneous flap. (B) Fabricated chimeric osteocutaneous flap. ALT, anterolateral thigh; LFCN, lateral femoral cutaneous nerve; OC, osteocutaneous; SCIA, superficial circumflex iliac artery. White arrow indicates distal continuation of the lateral circumflex femoral vessel. Black arrow indicates the vessel pedicle of the osteocutaneous flap. end corresponding to the arch of the foot. Fixation with a plate and screw was performed, and Kirschner $(\mathrm{K})$ wires were used when needed. End-to-end vascular anastomosis was then performed.

\section{Video 1}

Bleeding of the outer surface of the iliac graft before the vascular pedicle was divided. Online content including video sequences viewable at: www.thieme-connect. com/ejournals/html/doi/10.1055/s-0037-1604341.

To create a fabricated chimeric iliac osteocutaneous flap, an ALT flap was harvested initially in the standard manner. Most of the soft-tissue defect was first covered with an ALT flap, and its pedicle was anastomosed with recipient vessels. Then, an SCIA osteocutaneous flap was harvested and inset as described above. The vessel pedicle of an SCIA osteocutaneous flap was further anastomosed to either a side branch of the descending branch of the lateral circumflex femoral vessel or its distal continuation (- Fig. 2B).

\section{Results}

Four patients who underwent treatment with SCIA iliac osteocutaneous flaps and eight patients with fabricated chimeric iliac osteocutaneous flaps (combined with ALT flaps) were identified. Of these patients, 10 were males and 2 were females. The median patient age at the time of reconstruction was 41 years. Based on conventional anatomical definitions, 10 defects were located in the foot and 2 were located in the ankle. According to a new subunit classification system for the foot and ankle, ${ }^{14}$ four defects involved one subunit, three involved two subunits, and five involved three subunits. Five patients underwent initial debridement and vacuum therapy prior to flap coverage because of severe damage caused by high-energy trauma. The primary tumor diagnosis was pigmented villonodular synovitis.

The iliac segment size ranged from $1 \times 3 \times 0.7$ to $3 \times 6 \times 1 \mathrm{~cm}$, and the skin paddle size of the iliac osteocutaneous flaps ranged from $1 \times 4$ to $8 \times 16 \mathrm{~cm}$. The median length of the SCIA pedicles was $5 \mathrm{~cm}$ (range, $3-6 \mathrm{~cm}$ ). The ALT flap size ranged from $5 \times 10$ to $11 \times 25 \mathrm{~cm}$ with a median length of the vascular conduits of $6 \mathrm{~cm}$ (range, $5-8 \mathrm{~cm}$ ). All flaps were approximately 5 to $7 \mathrm{~mm}$ thick after primary debulking. Bone fixation was performed with plates and screws $(n=10), \mathrm{K}$ wires $(n=1)$, and screws $(n=1)$. A split-thickness skin graft was required to close one ALT donor site. The patient demographics and the surgical details are presented in - Table 1.

The distal portion of one ALT flap developed necrosis because of inadequate circulation caused by thinning; this complication was resolved by creating a filleted hallux flap. One SCIA osteocutaneous flap had to be revised 1 day after surgery because of vein thrombosis. After the thrombus was removed, reanastomosis was performed. Marginal necrosis 


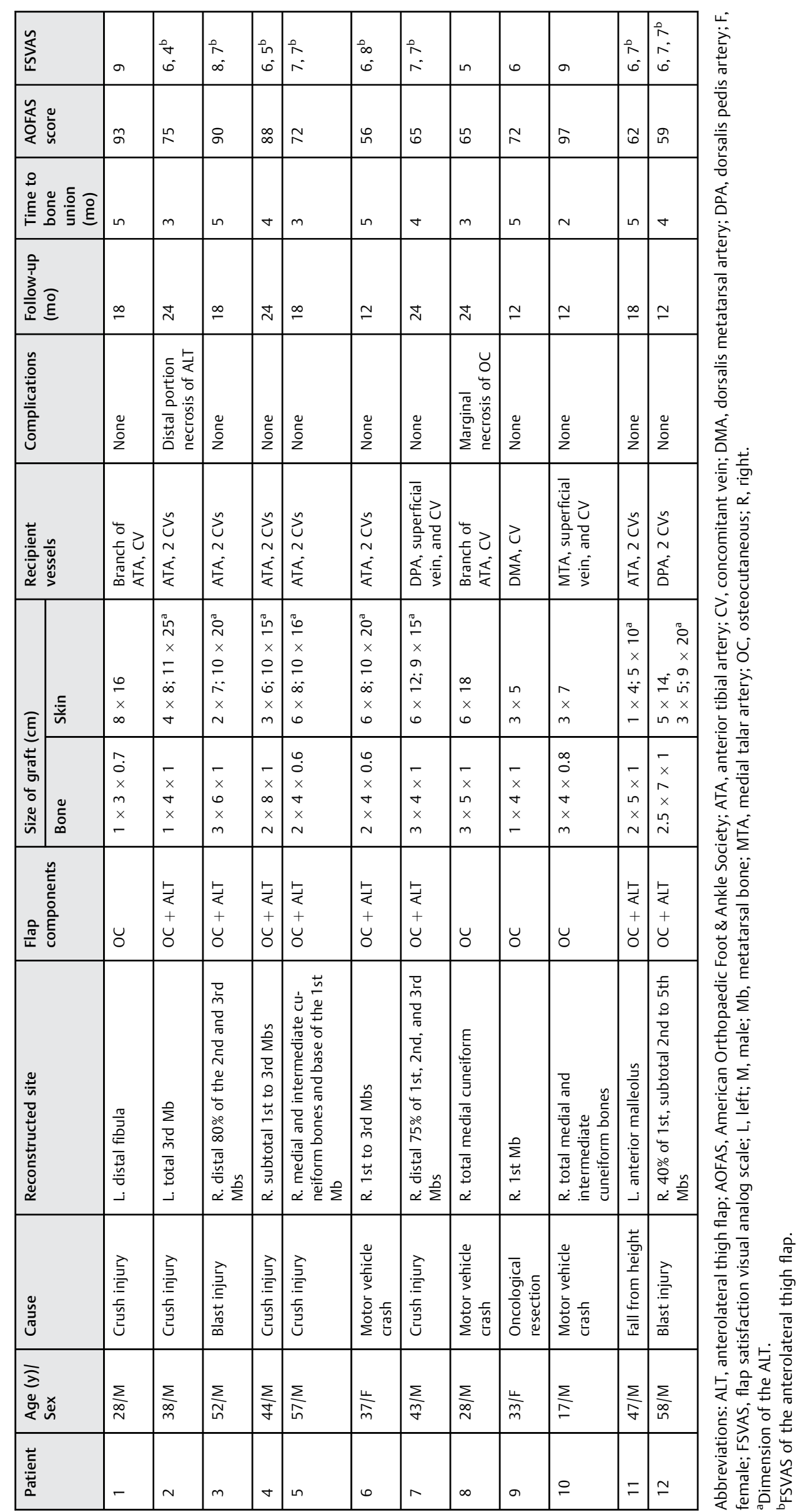




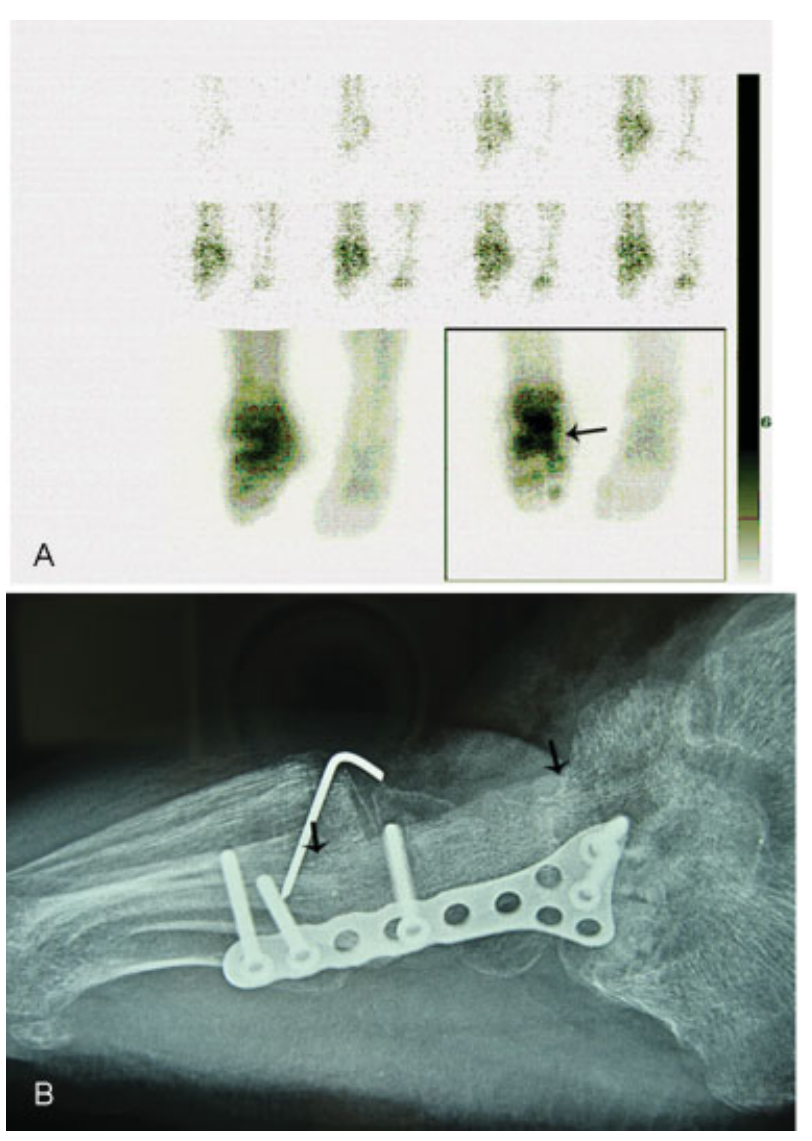

Fig. 3 A patient who sustained a total medial cuneiform bone defect. (A) Bone scintigraphy displayed viability by virtue of increased tracer uptake 11 days after reconstruction (black arrow). (B) Lateral radiograph obtained 3 months after surgery; union is noted at the site of arthrodesis (black arrows indicate the transplanted iliac segment).

occurred, which required a skin graft. However, the normal bone healing process was not affected (-Fig. 3A and 3B). All other flaps survived completely. The median follow-up time was 18 months. Nine implants were removed, and two ALT flaps were simultaneously debulked. No late flap instability was observed. The median time to bone union was 4 months. All patients regained full weight-bearing ability at a median time of 6 months. No stress fractures were observed. Remodeling and hypertrophy of the transplanted iliac segment were observed in three patients who had undergone metatarsal bone reconstruction. The patient who underwent tumor resection showed no sign of tumor recurrence. All patients were able to walk in normal footwear. No limitations in daily activities were reported. The AOFAS scores and VAS satisfaction scores regarding the flap at the last follow-up are presented in - Table 1. The scars at the donor sites healed well without pain.

\section{Case Reports}

\section{Case 1}

A 28-year-old male patient had been involved in a machine injury on his left ankle and distal lower leg ( - Fig. 4A and 4B). After fracture fixation of the distal tibia, the fibular defect was reconstructed with the iliac segment of an SCIA iliac osteocutaneous flap, and the soft tissue defect was covered with the skin paddle of the osteocutaneous flap (-Fig. 4C). The clinical presentation showed good contour and ankle function at 18 months after surgery (-Fig. 4D and 4E).

\section{Case 2}

A 43-year-old male patient had been involved in a traffic accident that caused a right foot crush injury. He had lost most of the soft tissue over the dorsum of the foot, including the distal $75 \%$ of the first, second, and third metatarsal bones (-Fig. 5A and 5B). The first metacarpal bone defect was reconstructed using the iliac segment of an SCIA iliac osteocutaneous flap, and the soft tissue defect was reconstructed using an ALT flap and the skin paddle of osteocutaneous flap (-Fig. 5C). The patient had an acceptable contour and foot function (-Fig. 5D and 5E).

\section{Discussion}

Previous reports have demonstrated favorable results using free vascularized osseous flaps for the reconstruction of foot and ankle bone defects. ${ }^{1-7}$ Favorable outcomes, when using this approach, are mainly associated with missing bone volume, amount of soft tissue available to cover exposed bone and tendon, and surgeon preference. For defects larger than $4 \mathrm{~cm}$, Bishop et $\mathrm{al}^{7}$ have advocated the use of a fibula flap, whereas a deep circumflex iliac artery-iliac crest flap is typically used for defects smaller than $4 \mathrm{~cm}$. However, it is important to consider the location of a skin perforator flap if bone is to be transferred with skin using such flaps. In the published data, the use of vascularized osseous flaps has not been exempted from major complications at harvest sites. Haddock et $\mathrm{al}^{1,5}$ favored the use of the medial femoral condyle for small bone defects (less than $3.5 \mathrm{~cm}$ ). However, the medial femoral condyle bone flap does not always have a convenient perforator supplying either the muscle or skin paddle, and thus, the use of a medial femoral condyle osteocutaneous flap, although possible in some patients, remains problematic ${ }^{15}$ and can leave a long scar on the medial aspect of the thigh. To the best of our knowledge, this report is the first case series describing the management of foot and ankle complex bone defects using an SCIA iliac osteocutaneous and fabricated iliac osteocutaneous flap.

Compared with traditional vascularized bone flaps, the major advantages of using an SCIA iliac osteocutaneous flap are easy accessibility from a well-concealed donor site and the ability to perform a unicortical, bicortical, or tricortical harvest, thereby reducing morbidity. An iliac bone segment can be raised with minimal soft tissue inclusion because the artery nourishing the harvested iliac segment originates from the periosteal branch of the deep branch of the SCIA. Another advantage is that it provides a relatively small- or medium-sized vascularized iliac bone flap along with a medium-sized thin perforator flap. This characteristic is suitable for reconstruction of complex defects in wounds involving the dorsal foot and ankle, since the use of a bulky flap may interfere with proper shoe fitting and prevent efficient ambulation. ${ }^{14}$ The blood supply from the SCIA to 

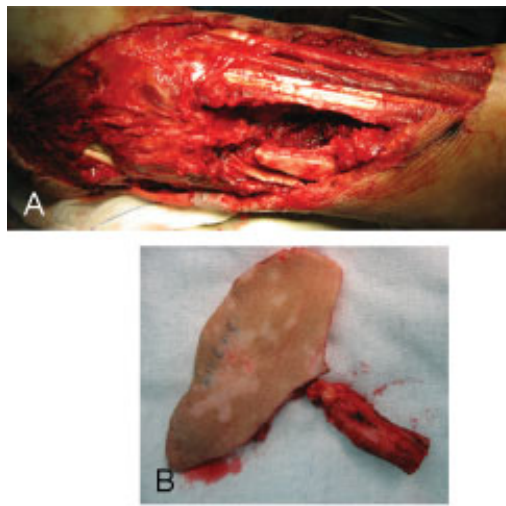
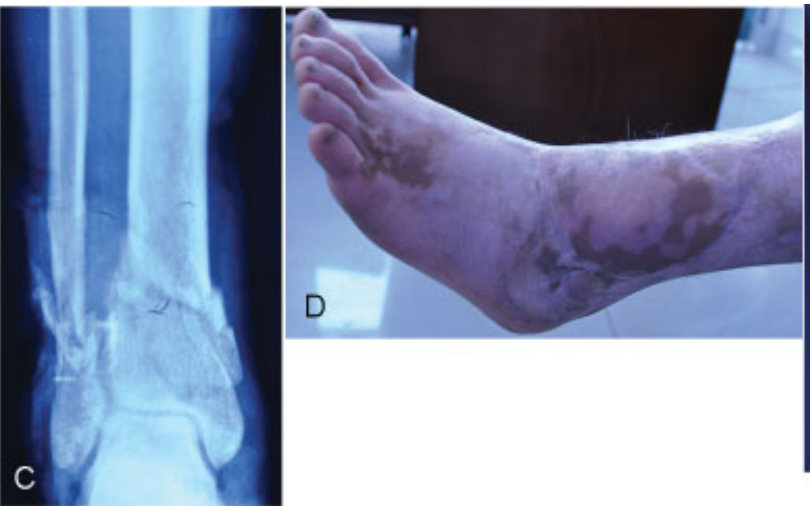

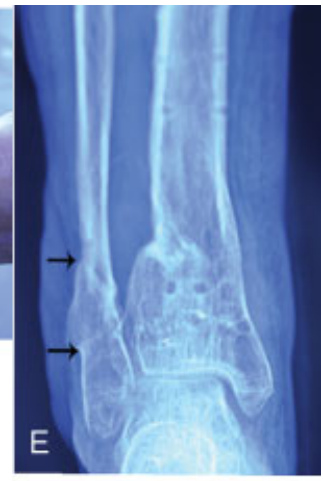

Fig. 4 Case 1: A patient suffered complex defect on his left ankle resulting because of a machine injury.(A) Preoperative clinical and (B) radiograph views. (C) The harvested osteocutaneous flap. (D) Appearance and (E) radiograph views after implant removal at 18 months after surgery (black arrows indicate the transplanted iliac segment).

the iliac bone is inferior to that of the deep circumflex iliac artery, which can be a problem when a long bone defect is being reconstructed. Taylor and Watson ${ }^{8}$ suggested that based on the SCIA, at least $8 \mathrm{~cm}$ of the iliac crest can be raised. In two cases of that series in which the bone segment length was $8 \mathrm{~cm}$, fresh bleeding was observed at the osteotomized sites before vascular pedicles were divided. Meanwhile, the maximum size of a transferred iliac segment also depends to some extent on its curvature. In our series, the iliac segment selected to reconstruct a bone defect was less than $8 \mathrm{~cm}$. The third advantage is that it can be fabricated as a chimeric osteocutaneous flap with an ALT flap. Multiple reports have shown that a medium-sized skin flap can be harvested based on the SCIA system. ${ }^{16,17}$ However, weaknesses associated with this procedure, such as a limited flap width affecting closure and a short pedicle length, limit its use for soft tissue defects that involve more than one subunit or are too large to be covered by a single SCIA osteocutaneous flap and in cases in which the pedicle length of the SCIA is inadequate for anastomosis. In these circumstances, we prefer to use a microsurgically fabricated chimeric flap. An
ALT perforator flap is our preferred additional flap because its thickness can be tailored to facilitate foot resurfacing. The descending branch of the lateral circumflex femoral artery can provide a suitable vascular conduit for the SCIA. ${ }^{18}$ However, in patients with a higher body mass index (greater than 23), an ALT flap will require secondary debulking to achieve a desirable contour. In this series, the maximum width of a skin paddle for an SCIA osteocutaneous flap was limited to $8 \mathrm{~cm}$ to permit primary closure, although Chao et $\mathrm{al}^{19}$ reported that the donor wound of an SCIA flap with a width less than $11 \mathrm{~cm}$ can generally be closed.

We acknowledge three main weaknesses in the current report. First, the number of patients is relatively small, and the patient characteristics are heterogeneous. This limitation should be considered when interpreting the results. Second, the position of a reconstructed metatarsal head is usually difficult to judge in cases of extensive longitudinal arch injury. To aid in determining the positions of the reconstructed metatarsal heads included in this series, the planes of the heel pad and the forefoot pad beneath the remaining metatarsal heads were measured using a large, sterilized
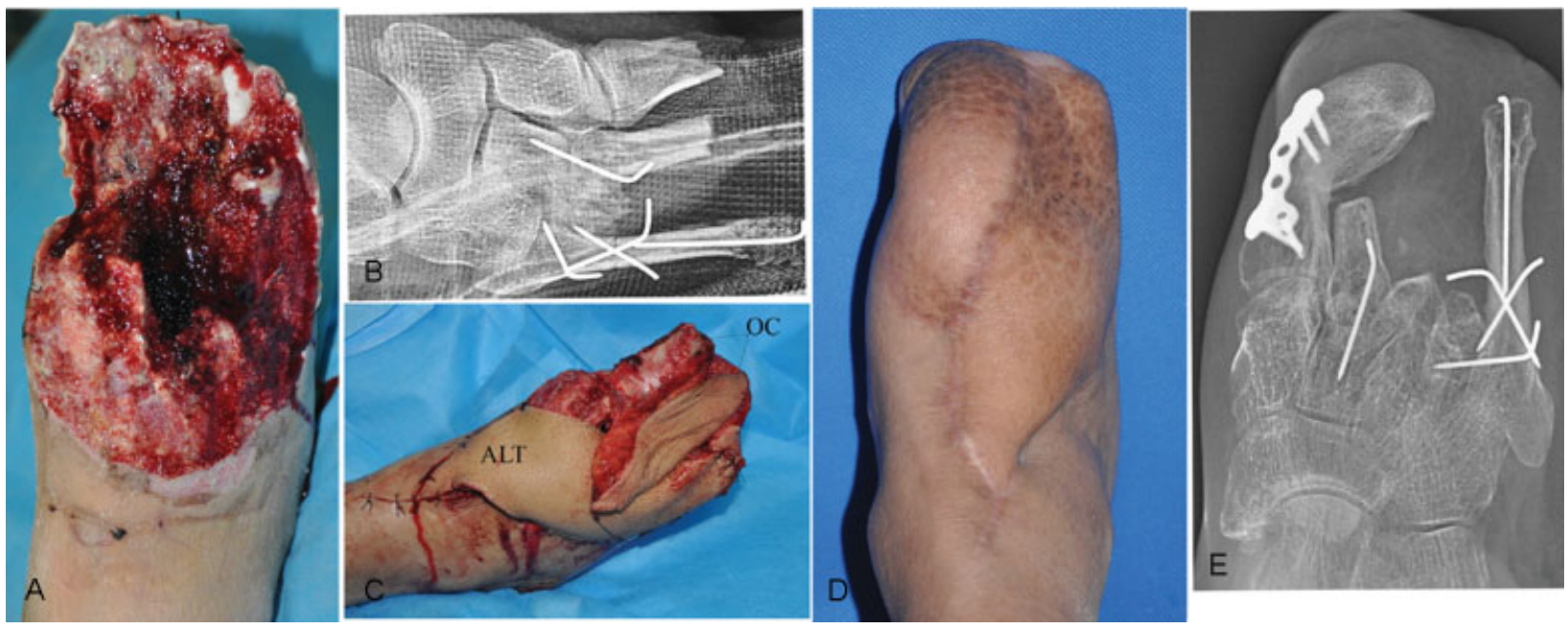

Fig. 5 Case 2: A patient suffered extensive loss of the dorsal skin of the foot, including most of the first, second, and third metatarsal bones, resulting because of a traffic accident. (A) Preoperative clinical and (B) radiographic views. (C) Appearance of the foot after both flaps were inset. (D) Appearance and (E) radiograph views at 24 months after surgery. ALT, anterolateral thigh; OC, osteocutaneous. 
square basin while the ankle was held in a neutral position. The injured metatarsal head could then be reconstructed to conform to this plane. The iliac segment was subsequently apposed to the greatest extent possible with the shaft of the remaining metatarsals. Third, this report did not include dynamic pedography evaluations to objectively analyze changes in gait and loading of the foot. The AOFAS score measures the overall ability of an individual to perform a list of activities specific to the affected foot; therefore, the values obtained may represent foot morbidity.

\section{Conclusion}

The use of free SCIA iliac osteocutaneous and fabricated chimeric iliac osteocutaneous flaps provides an alternative for treating small- and medium-sized bone defects (smaller than $8 \mathrm{~cm}$ ) along with soft tissue defects in the foot and ankle region. These flaps offer several advantages, including easy size adjustment, an acceptable contour, and low donor-site morbidity.

\section{Funding \\ None. \\ Conflict of Interest \\ None.}

\section{References}

1 Haddock NT, Wapner K, Levin LS. Vascular bone transfer options in the foot and ankle: a retrospective review and update on strategies. Plast Reconstr Surg 2013;132(03):685-693

2 Sahin I, Nisanci M, Aykan A, Guzey S, Ozturk S, Isik S. Reconstruction of the one-digit foot with an osteocutaneous fibula flap: a case report. J Am Podiatr Med Assoc 2014;104(05):526-530

3 Chuang DC, Chen HC, Wei FC. Technique of foot lengthening and shaping with free vascularized iliac osteocutaneous flap. Plast Reconstr Surg 1992;90(03):492-496

4 Roll C, Prantl L, Nerlich M, Kinner B. Osteo-fasciocutaneous parascapular flap transfer for reconstruction of the first ray of the foot. Arch Orthop Trauma Surg 2008;128(08):857-863

5 Haddock NT, Alosh H, Easley ME, Levin LS, Wapner KL. Applications of the medial femoral condyle free flap for foot and ankle reconstruction. Foot Ankle Int 2013;34(10):1395-1402
6 Clements JR, Mierisch C, Bravo CJ. Management of combined soft tissue and osseous defect of the midfoot with a free osteocutaneous radial forearm flap: a case report. J Foot Ankle Surg 2012;51 (01):118-122

7 Bishop AT, Wood MB, Sheetz KK. Arthrodesis of the ankle with a free vascularized autogenous bone graft. Reconstruction of segmental loss of bone secondary to osteomyelitis, tumor, or trauma. J Bone Joint Surg Am 1995;77(12):1867-1875

8 Taylor GI, Watson N. One-stage repair of compound leg defects with free, revascularized flaps of groin skin and iliac bone. Plast Reconstr Surg 1978;61(04):494-506

9 Iida T, Narushima M, Yoshimatsu H, YamamotoT, Araki J, Koshima I. A free vascularised iliac bone flap based on superficial circumflex iliac perforators for head and neck reconstruction. J Plast Reconstr Aesthet Surg 2013;66(11):1596-1599

10 Pan ZH, Jiang PP, Xue S. Free iliac flap for treating multiple skin defects of the hand and digits. J Hand Surg Eur Vol 2013;38(09): 952-958

11 Song B, Chen J, Han Y, et al. The use of fabricated chimeric flap for reconstruction of extensive foot defects. Microsurgery 2016;36 (04):303-309

12 Kitaoka HB, Alexander IJ, Adelaar RS, Nunley JA, Myerson MS, Sanders M. Clinical rating systems for the ankle-hindfoot, midfoot, hallux, and lesser toes. Foot Ankle Int 1994;15(07):349-353

13 Thankappan K, Kuriakose MA, Chatni SS, et al. Lateral arm free flap for oral tongue reconstruction: an analysis of surgical details, morbidity, and functional and aesthetic outcome. Ann Plast Surg 2011;66(03):261-266

14 Hollenbeck ST, Woo S, Komatsu I, Erdmann D, Zenn MR, Levin LS. Longitudinal outcomes and application of the subunit principle to 165 foot and ankle free tissue transfers. Plast Reconstr Surg 2010; 125(03):924-934

15 Rysz M, Grabczan W, Mazurek MJ, Krajewski R, Grzelecki D, Ciszek B. Vasculature of a medial femoral condyle free flap in intact and osteotomized flaps. Plast Reconstr Surg 2017;139(04):992-997

16 Yoshimatsu H, YamamotoT, Hayashi A, Iida T. Proximal-to-distally elevated superficial circumflex iliac artery perforator flap enabling hybrid reconstruction. Plast Reconstr Surg 2016;138(04): 910-922

17 Feng S, Xi W, Zhang Z, et al. A reappraisal of the surgical planning of the superficial circumflex iliac artery perforator flap. J Plast Reconstr Aesthet Surg 2017;70(04):469-477

18 Pan ZH, Jiang PP, Xue S, Li H, Wang JL. Restoration of basic hand function following devastating hand injuries using a microsurgically fabricated chimeric iliac osteocutaneous flap. J Plast Reconstr Aesthet Surg 2017;70(06):723-728

19 Chao WN, Tsai CF, Wang PH, et al. Freestyle groin flaps: the real axial flap design and clinical application. Ann Plast Surg 2015;74 (Suppl 2):S75-S79 